\title{
Simulated intensified images
}

\author{
Carlos Dorronsoro ${ }^{1}$, Deitze Otaduy ${ }^{1}$, Yolanda Navarro ${ }^{1}$ Javier Portilla $^{2}$ \\ ${ }^{1}$ Centro de Investigación y Desarrollo de la Armada, Madrid, Spain \\ \{cdorronsoro, dotaduyp, ynavher\}@oc.mde.es \\ ${ }^{2}$ Visual Information Processing Group, Dept. of Comp. Science and Artif. Intell., \\ Universidad de Granada, Spain \\ javier@decsai.ugr.es
}

\begin{abstract}
The most straightforward way to describe the performance of an image intensifier tube, especially under adverse conditions, is to predict the image it yields. In this work we have developed two different methods to provide realistic simulated images in low light level conditions: 1) Approximate Physical Model. A classical approach based on the simulation of the different degradation sources. It provides a good understanding of the image formation process. 2) Synthesis-by-analysis of real images. The observed noise is modelled through texture analysis tools and the image blur through the MTF. The resulting simulated images for both methods were compared with real intensified images (laboratory chart sights and natural images) taken under controlled conditions, close to the performance limits of an image intensifier tube. Both methods generated good results in terms of visual comparison for different object sizes, contrasts or luminances. These methods can be used as a new tool to predict the performance thresholds of the image intensifier. Only well-known or measurable parameters were used as input for the methods.
\end{abstract}

Keywords: synthetic images, simulated images, image intensifier tubes, intensified images, MTF, texture synthesis, Low light level, Night Vision, Minimum Resolvable Contrast.

\section{INTRODUCTION}

Image Intensification is a widely used technology when working in low light level conditions. The applications range from night vision in the visible or near infrared to medical or scientific devices operating in different regions of the electromagnetic spectrum.

Image intensifier (II) tubes are frequently used near their performance limits. Quite often critical tasks such as driving, piloting, surveillance and threat detection, medical diagnosis, tracking of nuclear particles or the imaging of astronomical bodies, rely heavily on the quality of the images delivered by the intensifier. Moreover, intensified cameras are increasingly being used for quantitative measurements. The selection of an II tube for a particular application or even the design of a complete imaging system based on image intensification is often a complicated task, since different compromises have to be achieved ensuring that the final performance of the system is not affected.

The general practice is to describe the performance of the II tube by means of a few parameters following military standards (gain, EBI, MTF, noise, resolution). These parameters describe only partially the characteristics of the II tube's response and are usually applicable merely to certain supra threshold conditions. Furthermore, even if the influence of each individual parameter on the final image is well known, the impact of a combination of them upon the image is far from being intuitive. Besides, the current industrial trend is to summarize the quality of the device in just one or two numbers, which is certainly insufficient for design purposes.

Regarding a more theoretical approach, various mathematical models have been proposed to provide a detailed description of the physics of the II tube and its components ${ }^{1,2}$. Some of these statistical models are helpful to understand 
how the II tube works or to predict some performance metrics values (for instance the overall gain ${ }^{3,4}$, limiting resolution, MTF, signal-to-noise ratio ${ }^{4,5}$, or halo ${ }^{6}$ ). Some of them are useful to quantify some design trade-offs of intensified CCD cameras: SNR versus spatial resolution ${ }^{4,5}$ or SNR versus gain ${ }^{4}$. Most of these physical models have the drawback of being too complex for practical purposes. Furthermore, many of them cannot be experimentally validated, since certain required parameters are neither known nor can be measured once the II tube is encapsulated.

In our opinion, the most straightforward way to describe the II tube's performance for a given situation (radiance field) is to predict the image yielded by the II tube. This would permit a direct comparison among different II tubes, systems, configurations or situations within the boundaries of the luminance range in which it is employed. Moreover, a visual comparison of images would be more intuitive for final users.

This paper aims at exploring new mathematical methods to simulate realistic images as they would be produced by a given II tube. Two different approaches will be described: 1) Approximate Physical Model: this represents the classical approach, based on the simulation of the various degradation sources, but using only known or measurable parameters as inputs; 2) Synthesis-by-analysis of images: this new global approach relies on the extraction of a set of significant image features. This set is subsequently employed to generate a realistic image having a similar visual appearance as the original one. To assess the efficacy of each model, the simulated images were compared with real images obtained with the intensifier under controlled conditions on a broad range of the object size, luminance and contrast.

Simulated intensified images have other potential applications for the evaluation and testing of intensified systems. Collections of real images, captured in field trials, are seldom used for a rigorous prediction of the performance of image intensifiers. First, it is hardly ever possible to have access to the object to be imaged. Second, even when the object is accessible, it is expensive and time consuming to collect a sufficient number of images under controlled conditions. Finally, the conclusions are limited to those specific sampled situations.

Laboratory trials -although still expensive, since tailored testing facilities are required- are more common to evaluate intensified imaging systems. The measurement of simplified objects (Minimum Resolvable Contrast) provides an indirect procedure to assess the detection, recognition or identification distance for well known targets (Static Range Performance). But it requires some simple modeling tools to extrapolate to real targets and to include atmospheric effects. This measurement and modeling have been used for many years with good acceptance by the military community. In fact, there is still ongoing research to improve the procedures. However, even for the specific purpose of calculating the Static Range Performance, the use of simulated images could provide a more direct and powerful method, specially in high-complexity scenes.

Traditionally, most II tubes were integrated in vision system, playing the role of vision aid for the observer. Nowadays an increasing number of intensifiers are coupled to artificial vision algorithms that extract the relevant information from the recorded image. These algorithms need to be refined and thoroughly debugged with the help of a large number of test images. In this context, simulated images can provide an unlimited number of source objects or scenes in a continuous range of situations.

There have been some previous attempts to generate simulated intensified images ${ }^{7}$. Most of them focus on supra threshold (luminance, contrast) conditions and are applicable only to ICCD cameras, in which the pixel-averaging mechanism alters the spatial structure of the image emerging from the II tube, both in terms of blur and noise. Finally, and as far as the authors are aware, none of them have been validated under controlled situations.

We think that our approach is a novel and innovative one for various reasons: 1 . We just simulate the image that would be delivered by the II tube (excluding the effect of the CDD); 2 . The simulations focus on the system's limit operating conditions (previous works assumed supra-threshold conditions); 3. Only well-known or measurable II tube parameters are used in the models; 4. These methods provide an overall characterization of the II tube in terms of image quality, instead of being restricted to specific aspects like the luminance response or the noise; 5 . The methods use the physical magnitudes of the scene; 6 . They are generic methods, although they can also be customized to specific applications; 7. They are simple models, which entail the assumption of certain approximations; 8 . The whole procedure is validated using a set of images recorded experimentally under controlled conditions (II tube-oriented metrology laboratory). 
The present work is structured as follows. Section 1.1 illustrates the operating principle of an image intensifier and the main characteristics and features of intensified images. Sections 2.1 and 2.2 describe the two methods implemented in this study for the generation of simulated images: the Approximated Physical Model and the Synthesis-by-Analysis of real images. Section 2.3 focuses on the experimental recording of real intensified images, which are compared to the results of the simulations in section 3. Section 4 analyzes the potential applicability of the models developed and implemented in this study.

\section{1. intensified images}

Incident photons at II tubes impinge upon the photocathode and a fraction of them are converted into photoelectrons. Electrons are accelerated across a potential difference onto the microchannel plate. Secondary electrons are created by the collision of the primary electron with the microchannel's walls. These secondary electrons emissions cascade down the length of the II tube. A second potential difference accelerates these electrons toward the phosphor screen, which fluoresces as a result of the collision. The outcome of the process is an amplified image output signal, due to the fact that around $10^{4}$ photons are generated on the phosphor screen for each photon captured by the photocathode ${ }^{5}$. This remarkably high amplification, which is described by the luminous gain factor, allows the image intensifier to generate images of very dim scenes (as star-light illuminated scenes). In these low-light-level conditions, radiation is affected by photon noise, which can be described by Poisson statistics. Every amplification stage propagates the existing noise according to its gain while incorporating an additional noise component (due to the non-uniformities of the gain). This noise propagation in image intensifiers is usually modeled as a cascaded system ${ }^{4,5}$. Despite its complexity, the global noise associated to the system is usually described by a single parameter: the Signal-to-Noise Ratio (or the Noise Equivalent Irradiance). On the other hand, the spatial response of image intensifiers can be described by means of the $\mathrm{MTF}^{8}$. Both the spatial blur and the finite integration time of the phosphor screen produce an overlapping of close events (in space or time) that reduces the overall noise in the intensified image. Noise and blur are the main causes of image degradation. Blur affects noise, as it changes its spatial correlation. Given that this work seeks to deliver an accurate prediction of intensified images, based on the visual impact of the degradation sources on the images, the abovementioned spatial correlation has to be taken into account (unlike other models for image synthesis, which make use only of first-order statistics).

\section{METHODS}

\subsection{Approximate Physical model (APM).}

The first part of this study was to develop a physical model capable of predicting image appearance under different situations. We were particularly interested in simulating images in limiting situations: luminance, contrast, spatial resolution near its threshold values, since in these particular regions existing models have been very little tested. Following a similar approach to other models ${ }^{7}$, the Approximate Physical model is based on the description of the various degradation sources as image filters. To guarantee the practical usefulness of the model, some approximations are required to ensure that we only include well-known and measurable parameters.

The model is described in figure 1 . Given the luminance distribution at the scene $L s c$, the resulting illuminance distribution at the photocathode can be calculated as follows:

$$
E p c=\frac{\pi \cdot L s c \cdot T a t m \cdot T o b j}{4 \cdot F \#^{2} \cdot(1+m)^{2}}(\mathrm{~lx})
$$

where

$$
\begin{aligned}
& L s c=\text { scene luminance distribution }\left(\mathrm{cd} / \mathrm{m}^{2}\right) \\
& F \#=\text { F-number of the system objective lens } \\
& \text { Tatm, Tobj = Atmosphere transmittance and objective lens transmittance } \\
& m=\text { Magnification of the optical sub-system preceding the II tube. }
\end{aligned}
$$

Once the illuminance distribution at the photocathode is obtained, the mean number of emerging photoelectrons is directly dependent on the photocathode's sensitivity: 


$$
<N_{e}>=\frac{\text { Epc } \cdot \text { Apix } \cdot S}{1.62 \cdot 10^{13}}
$$

Where $S$ is the photocathode's sensitivity (this is the only parameter included in the simulation that is related to the II tube specifications provided by the manufacturer), Apix represents the pixel area, as measured at the photocathode's plane $\left(\mathrm{m}^{2}\right)$ and $N_{e}$ indicates the average number of photon-generated electrons at the photocathode per unit time and per pixel. Besides photoelectrons, the spontaneous emission of electrons by the photocathode also needs to be taken into consideration. This phenomenon can be modeled by means of an additional illuminance factor, which is usually wrapped in a parameter commonly known as EBI (Equivalent Background Input).

From here onward no further distinction will be made between photoelectrons and spontaneously-emitted electrons. We will consider the overall electron emission to follow a Poisson-type distribution, for which the variance of the number of photons in each point is equal to the average number of photons.

$$
\sigma^{2}=<N_{e}>
$$

In practice, once the image has been generated (in terms of the average number of electrons $\langle N e\rangle$ in each point), a random deviation following a Poisson distribution is applied to each individual value.

As previously mentioned, the electrons emerging from the photocathode multiply in the II tube through various mechanisms and in several stages before they are converted again into photons. Each of these processes introduces an additional noise component due to the non-uniformity inherent to the amplification procedure, as well as a certain degradation in image quality due to the widening of the electron beam along the II tube path. While the amplification process can be described by means of the gain $(\mathrm{G})$, the overall noise is characterized by the value of the signal-to-noise ratio (SNR) and the spatial image degradation is quantified by means of the modulation transfer function (MTF). However, these three parameters are highly coupled in II tubes: the value of the SNR depends on G and it is also affected by the MTF. A rigorous approach would imply the assessment of the gain, the noise and the MTF separately and on an element-by-element basis. For instance, the gain amplifies the resulting noise from previous stages, whereas the MTF of each stage reduces all the existing noise. Nonetheless, and since our approach aims at including only well-known or measurable II tube parameters, this model is limited to using global II tube values for G, MTF and SNR, which represents a considerable simplification compared to the element-by-element approach.

Considering that our simulations focus on low luminance levels, a constant gain factor, equal to the maximum value of the gain for that particular II tube, has been assumed. This assumption, which is equivalent to saying that the automatic gain control remains inactive, has been later backed by our experimental results (figure 4, series 1). This way, the electron count for each point can be directly multiplied by this constant gain factor in order to assess the resulting number of photons or digital counts. A measurement of the overall gain (defined as digital counts to input illuminance ratio) has been used instead of a luminous gain factor (defined in STANAG standards as output luminance to input illuminance ratio), because this work aims at focusing on a quantitative analysis.

There are other noise sources affecting the final image quality, besides Poisson-related noise. Measuring the SNR while adhering to STANAG standards is a complex experimental task and its final result is subject to a high uncertainty. Moreover, practical experience has shown that the amount of spatial noise varies according to the square root of the luminance. Therefore, for the present application we have opted to make use of a simpler magnitude, the Noise Factor $(\mathrm{NF})$, which experimental measurement is of a more robust nature, and makes the noise parameter independent of the luminance. The noise factor used in this study is defined as the existing standard deviation $(\sigma)$ divided by the square root of the average signal value.

$$
N F=\frac{\sigma_{\text {signal }}}{\sqrt{\text { signal }}}
$$

The Poisson distribution has a noise factor equal to one. The final image has more noise and subsequently a Noise Factor greater than one. The spatial correlation introduced by the MTF reduces the noise factor of the image. In this paper, the 
Noise Reduction Factor (NRF) produced by the particular MTF of the II tube is previously measured on simulated Poisson images, with uniform luminance.

$$
N R F=N F(\text { Poisson }, M T F)
$$

After that, the II tube noise is added to the Poisson noise as a multiplicative Gaussian distribution. Its sigma value is calculated based on the local luminance, the Noise Reduction Factor (due to the II tube's MTF) and the measured Noise Factor itself:

$$
\sigma_{\text {tube }}=\frac{N F}{(N R F \cdot \sqrt{L L})}
$$

where $L L$ is the local luminance.

That is, the value of the multiplicative noise factor in each point associated to the existing electrons is high compared to the noise present in the final image, due to the fact that the MTF-related noise reduction has already been taken into account. Finally, the MTF is applied to the final image in the form of a linear filter.

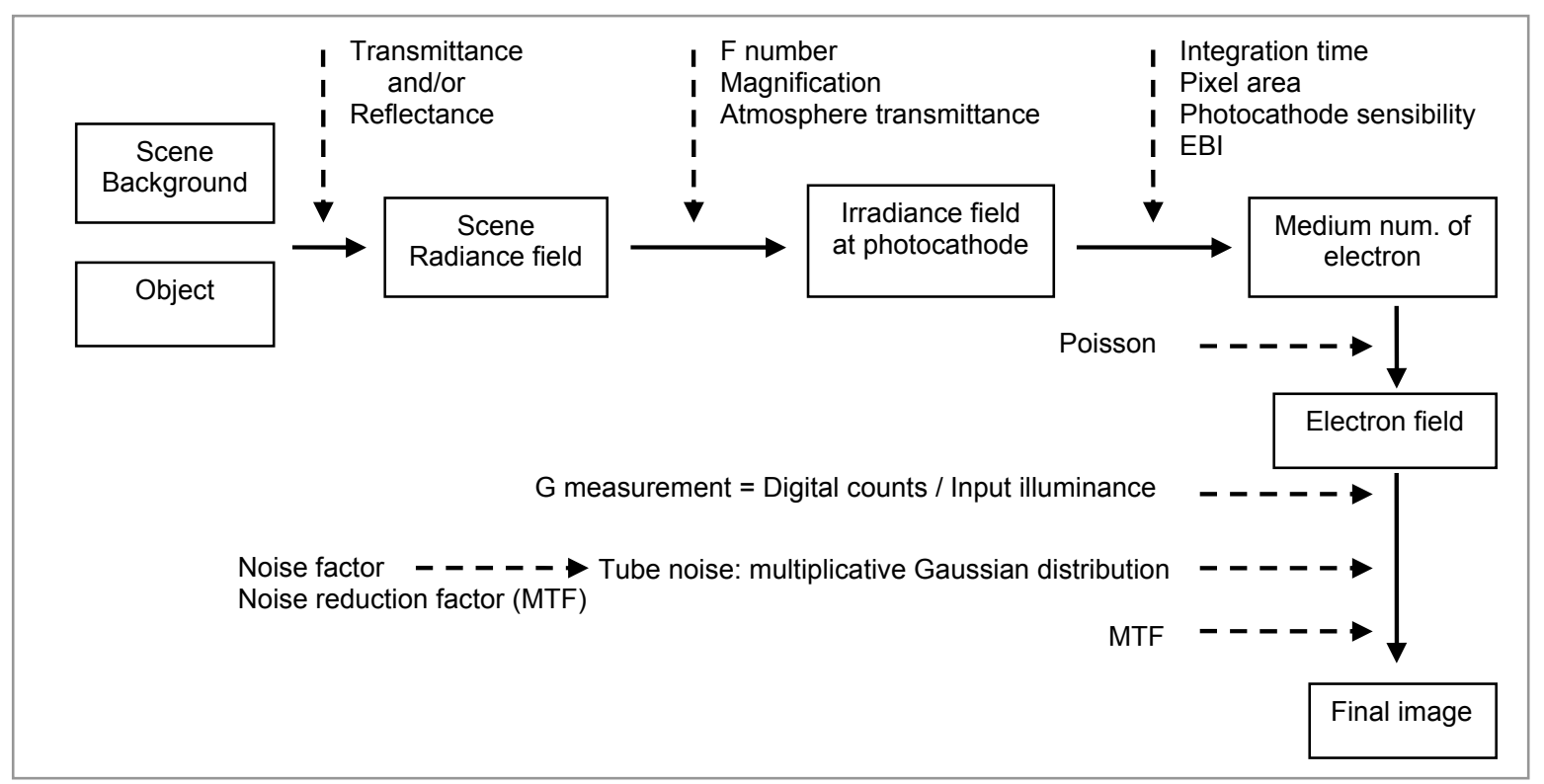

Figure 1. Approximate Physical Model (APM)

\subsection{Synthesis-by-analysis of images method (SBA)}

The Approximate Physical Model described above includes many approximations, and depends on a large number of parameters, which can be inaccurate or not valid for the whole luminance range. That is why a new method was developed, in order to avoid the dependence on approximations or parameters.

This completely different approach relies on a previous analysis of a particular set of real images in order to extract the necessary descriptors that will be used during the subsequent synthesis stage. As shown in figure 2, the observed noise is modeled using existing texture-analysis tools whereas the image blur is computed based on the measured global MTF ${ }^{8}$. The final simulated image is the result of blurring the initial image by applying the measured MTF and subsequently adding the synthetic-texture modeled noise. This heuristic approach provides a black box in between the luminance distribution of the scene and the final image. No further assumptions regarding how the real image is created are required. 


\subsubsection{Texture Synthesis-by-analysis}

A simplified texture synthesis-by-analysis tool based on Gabor functions (Portilla et al. ${ }^{9}$ ) was used to generate perceptually-accurate synthetic images.

The synthesis-by-analysis methods rely on the extraction of a set of significant image features, which are subsequently employed to generate a realistic image having a similar visual appearance as the original one. There are various texturesynthesis methods available in the literature, ranging form those purely statistical methods to the more structured ones. Ideally, a texture synthesizer should fulfill the following requirements: wide range of applications, compactness, low computational cost, full automatism and robustness. Several successful methods have employed the approach of analyzing the texture's autocorrelation function, to be subsequently imposed to a random 2-D signal. However, these algorithms present important drawbacks; for instance, they show a slow convergence (which implies that the final image presents a noisy aspect) and they are very time-consuming (high computational cost). These disadvantages can be minimized only if one or more significant parameters is fixed in advance, although this step is quite problematic to automate.

The approach followed by Portilla et al. ${ }^{9}$ consists of sampling the autocorrelation function through a multiscale Gabor representation. This sampling type has proven to be compact and visually efficient, as it is based on a priori information about the behavior of the human visual system. Furthermore, this method has shown to be robust, it uses noniterative simple operations as linear filtering and histogram matching, and provides good results over a wide range of input textures. This model focuses on nonstructured, gray-level textures. Texture is modeled as a 2-D random field, which enables a purely-statistical treatment.

The Multiscale Gabor Scheme ${ }^{10}$ schematically imitates the visual coding in the early stages of the HVS by applying a set of $4 \times 4$ (four frequency levels, four orientations) Gabor filters to the digital images. This scheme is suitable for a fast pyramidal image decomposition in both the spatial, as well as in the spatial-frequency domains. The result of applying one of these filters to the input image is called a Gabor channel. The very low-spatial-frequency region of the image is covered by an additional low-pass residual (LPR) channel. This original configuration has been maintained for the present work as a precaution against possible directional structures in the image. However, so far the sampled II tubes have shown a complete absence of directional structures, which could imply that a unique average orientation might be sufficient. Regarding the four different radial frequencies, they have proven to be well-suited to capture all the relevant information for the textures resulting from the spatial noise associated to the II tube-generated images.

The energy of each of these 16 channels, together with a compressed version of the modulus of the LPR channel (5 parameters), provide a rough approximation to the power spectral density of the texture, although it has proven to be visually a very efficient one. The equivalent bandwidths of the channels, used in the original study ${ }^{8}$ was not considered here due to the stochastic nature of the noise.

Unlike the original work by Portilla et at. ${ }^{9}$, where a compressed version of the 256 -gray-level histogram (16 values) was included as well in the model, here the image histogram is simply fitted to a Gamma-function described by only 2 parameters. The reason behind the choice of a Gamma function instead of a Gaussian distribution (as in the original work) is that the histogram for images generated by II tubes shows a certain luminance-dependent kurtosis. As a consequence, the output image's histogram doesn't follow a Poisson distribution (unlike it happened with the impinging photons) and the assumption of a Gamma distribution has proven to yield a better fitting. To improve the efficiency of the subsequent synthesis stage (histogram fitting), three additional parameters are included in the model to describe the range, in terms of digital counts, in which the texture is found. In summary, the texture under analysis is characterized by just 26 parameters: energy of each Gabor channel (16) + LPR (5) + Histogram (5).

The basic idea behind texture synthesis is to employ the result of the original texture analysis (summarized in the abovementioned 26 parameters) as source data in order to generate secondary textures having the same visual appearance as the original one. In this sense, synthetic Gabor channels are generated, similarly to those obtained for the original texture, and through their fusion the new texture is obtained. 2-D noise following a Gamma probability distribution is generated, to which texture analysis is applied in order to obtain 16 noise channels. Each of these channels is multiplied by a certain factor in order to make the channel's energy the same as that for the corresponding channel in the original 
texture. The 16 synthetic channels and the LPR are mixed together into a single image. The original histogram (the Gamma function described by its parameters) is imposed to the resulting image.

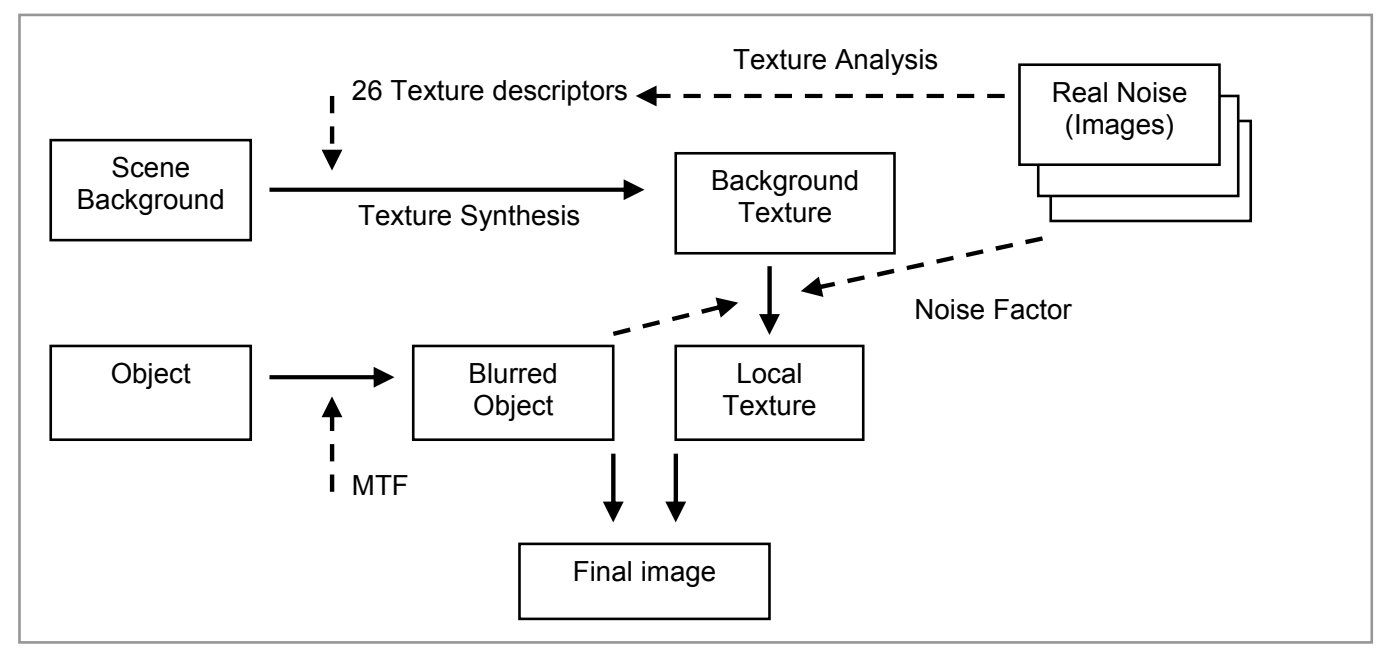

Figure 2. Synthesis by Analysis of images (SBA)

\subsubsection{Image generation}

In II tubes there is a strong dependence of the noise's intensity on the scene's luminance. Therefore, the simple addition of the texture to the MTF-degraded image doesn't yield satisfactory results. The texture needs to be modulated, as described below.

II tubes show a very large interscene dynamic range $\left(10^{6}\right)$, along which the noise varies significantly both in power and spatial statistics. In this sense, figure 3 shows the considerable variation of the texture descriptors within the II tube's dynamic range, both for a GEN-II and for a GEN-III tube. Texture analysis need to be carried out for different luminance levels. Texture synthesis should be based on texture descriptors for a particular luminance level, which can be interpolated from the analyzed luminances.

The intrascene dynamic range is typically less than 10, specially near the II tube performance limits where the image contrast is low. As can be seen in figure 3, the texture descriptors present a slow evolution inside a scene. The fundamental assumption in this method is the fact that the spatial noise in any intensified image can always be described in terms of a single texture (a unique set of parameters). This simplifies its practical implementation. But, as the amount of spatial noise varies according to the square root of the luminance, the noise's standard deviation varies substantially across a given scene -the local histogram changes-. To take this fact into account the texture's standard deviation is modulated by the luminance value at each point, to achieve the standard deviation corresponding to the noise factor:

$$
\sigma_{\text {target }}=N F \cdot \sqrt{L L}
$$

where $L L$ represents the local luminance. The Noise Factor $(N F)$ is obtained empirically during the synthesis stage. Those points which local luminance is equal or below that of the scene's background will remain unaltered. According to equation (7), the higher the local luminance, the higher the associated spatial noise. In practice, the modulation is applied by:

$$
M T=(T-\bar{T}) \cdot \frac{\sigma_{\text {target }}}{\sigma_{\text {texture }}}+\bar{T}
$$

where $M T$ is the modulated texture, $T$ is the synthetic texture which mean value is $\bar{T}$ and standard deviation is $\sigma_{\text {texture }}$. 
The object is degraded by the MTFs of optics and II tube. And finally, the obtained modulated texture is added to it (figure 2).

GEN III

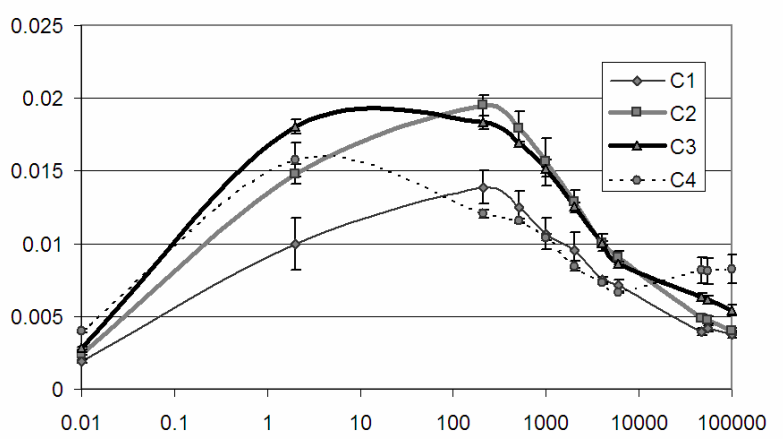

GEN II

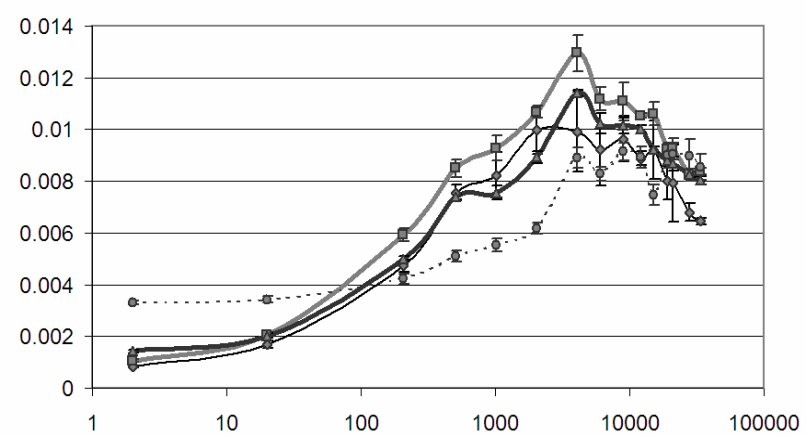

Figure 3. Radial texture descriptors along the full dynamic range (in arbitrary units) for two image intensifiers.

\subsection{Image capture}

Real images of various objects were captured to be used as reference, enabling benchmark testing of the simulated images. Additional images of the luminance background having no object were recorded to obtain the values of the input parameters required for the simulations: signal-to-noise features as well as texture descriptors. The II tube used to record these images was a GEN III (ITT F9800P) mounted on a monocular night vision sight (ITT PocketScope). The lowluminance source was specifically designed to supply a uniform and stable spectrum having a color temperature of $2856 \pm 20 \mathrm{~K}$ (calibrated following European standards). Three calibrated luminance positions were used $\left(\mathrm{L}_{1}=7 \cdot 10^{-3}\right.$; $\left.\mathrm{L}_{2}=7 \cdot 10^{-4} ; \mathrm{L}_{3}=6.7 \cdot 10^{-5} \mathrm{~cd} / \mathrm{m}^{2}\right)$, equivalent to having from a quarter-moon to a clouded-star-light illuminated scene. The radiance field of the scene was simulated by placing grey-scale images on $6 \times 6$-cm slides in front of the source and projecting them onto the night vision sight objective $(3 \mathrm{x}, \mathrm{F} \# 1.5)$ by means of a collimator (silver-coated off-axis paraboloid mirror, focal length $2496 \mathrm{~mm}$, diameter $430 \mathrm{~mm}$ ). The ocular of the night vision sight was removed and the phosphor screen was imaged by means of a CCD (Hamamatsu ORCA 100, pixel size 6.7 x $6.7 \mu$, Peltier cooled, 12 bits). The camera is lens coupled $(1: 1.7)$ using an $85 \mathrm{~mm}$ photographic lens (Nikon) followed by an inverted $50 \mathrm{~mm}$ photographic lens (Nikon). This configuration was selected because it acts as a low magnification microscope, providing a good trade-off between image quality and light capture. The camera readout noise is well below the events of the intensified images even for the lowest luminance scene. Only the central 256 x 256 pixels of the image were used, subtending $1.01 \times 1.01 \mathrm{~mm}$ of the phosphor screen, where its curvature can be assumed to be negligible. The following parameters were previously measured (or checked) in our laboratory following procedures adapted to international standards: luminance stability, luminance uniformity, luminance level, source spectral response, target spectral transmission, target contrast, linearity and uniformity of the camera, II tube gain, linearity, MTF, EBI, and signal-tonoise ratio.

\section{RESULTS}

Figure 4 shows the first-order statistics for the images yielded by the II tube when imaging a series of uniform-luminance scenes of different luminance values $\left(\mathrm{L}_{1}, \mathrm{~L}_{2}\right.$ and $\left.\mathrm{L}_{3}\right)$. Series 1 (represented on the graph by rhombuses) shows the mean value across the image (in digital counts) captured by the camera. As can be inferred from the graph, the II tube shows a completely linear behavior within this luminance range. EBI and residual light are negligible. The automatic gain control of the II tube, as could be expected, is not active, taking into account that only the central region of the II tube is illuminated (only a few millimeters wide). Hence, the II tube is working in its full-gain regime. This high linearity ensures that we can consider a unique global gain factor in between the luminance of the scene and the (mean) response of the camera in digital counts. This factor is obtained by carrying out a linear fitting to the data points of series 1 . It is the overall gain factor used in the APM. Series 2 (represented by the squares) shows the standard deviation of each of the three real images. Series 3 (represented by the triangular symbols) shows the ideal Poisson noise. A noise factor of 3.117 is obtained for this II tube from the linear fitting of the series- 2 data, which, in other words, means that the final noise in 
real images is 3.117 times higher than the corresponding Poisson noise for each luminance. This value is included in both simulation methods $(6,7)$ described above to quantify the noise introduced by the II tube. Finally, Series 4 (represented by the circles) shows the expected standard deviation in the image if only Poisson noise and MTF were present. The spatial correlation of the noise produces a significant fall in the standard deviation of the signal, as expected. The noise factor decreases from 1 (Poisson, Series 3) to 0.044 (Correlated images, Series 4). Hence, the value 0.044 is the Noise Reduction Factor (5) that needs to be introduced into (6) to calculate the multiplicative Gaussian distribution that models the noise introduced by the II tube, before the spatial correlation caused by the MTF.

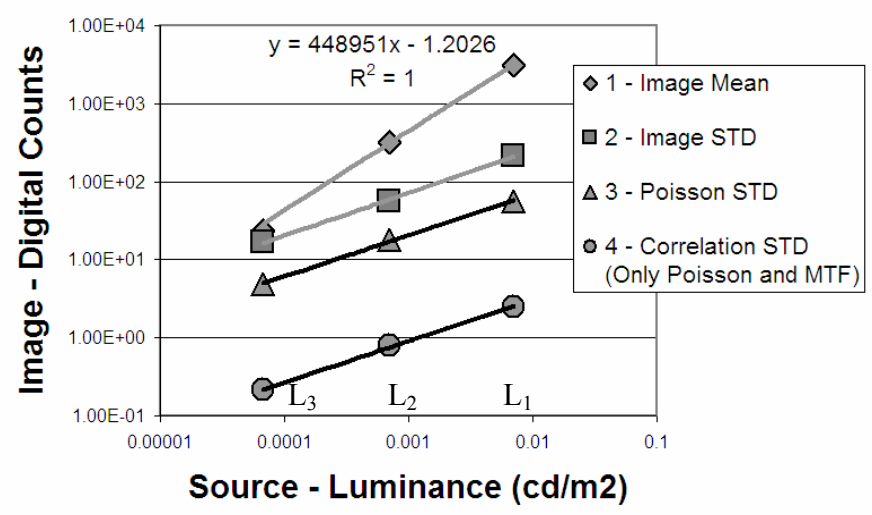

Figure 4. First-order statistics for the real images. See text for a detailed explanation.

In terms of visual comparison, we have summarized in figure 5 the resulting images of the simulations using both methods described above as well as the images experimentally recorded in our laboratory under controlled conditions. In all cases the object was an USAF 1951 target (left column). The "REAL" column shows the images experimentally captured in the laboratory following the methods described in section 2.3. The simulated images as yielded by the Approximate Physical model are located on the "APM" column, whereas the ones simulated following the Synthesis-byAnalysis method are on the "SBA" column. Rows 1 to 3 corresponds to decreasing levels of background luminance $\left(\mathrm{L}_{1}\right.$ to $\mathrm{L}_{3}$ ) of an USAF 1951 sight with $99 \%$ Michelson contrast [(Lmax-Lmin)/ (Lmax+Lmin)]. Rows 5 and 6 correspond to a contrast of $25 \%$ and luminances $\mathrm{L}_{1}$ and $\mathrm{L}_{2}$. In this bench, the biggest sight of the image (Group 0, Element 1) has an angular frequency of 2.5 line pairs per milliradian. Consequently, even with the best contrast and luminance, the night vision sight is operating close to its performance limit, since its measured angular resolution is of 6.3.

Figure 6 follows a similar layout as figure 5, but in this case the object was a tank which luminance distribution (or slide transmittance distribution) is shown in the left column. Column "REAL" shows the real image emerging from the II tube while columns "APM" and "SBA" shows the results for the simulations. Rows 1 and 2 correspond to decreasing luminance levels $\left(\mathrm{L}_{1}\right.$ and $\left.\mathrm{L}_{2}\right)$. Rows 3 and 4 correspond to a tank of half size (simulating double distance).

Regarding the values included in the two simulation methods, the input parameters used in the APM are shown in table 1, whereas the SBA method makes use of the noise factor (measured on the same images), the measured MTF, the transmittance distribution of the object, the background luminance, and a set of 26 input parameters describing the texture (obtained from the images of uniform luminance, see 2.2.1). As the background luminance is exactly the same for the laboratory images and for the simulated images, no interpolation on the parameters describing the texture is required here.

Table 1. Approximate Physical Model input parameters.

\begin{tabular}{|l|l|l|}
\hline Bench & Il tube & Object \\
\hline $\mathrm{F} \#=1.5$ & $\mathrm{~S}=1800$ & Object transmittance distribution \\
$\mathrm{T}_{\text {obj }}=\mathrm{T}_{\text {collimator }} \cdot \mathrm{T}_{\text {objective }}=0.95 \cdot 0.90$ & $\mathrm{EBI}=2.5 \mathrm{e}-7$ & Background luminance \\
$\mathrm{Tatm}=0$ & Measured MTF & Integration time $=111 \mathrm{~ms}$ \\
$\mathrm{~m}=\mathrm{f}_{\text {obj }} / \mathrm{f}_{\text {collimator }}=80 / 2497$ & Noise Factor $=3.117$ & \\
Apix $=(6.7 \mathrm{e}-6 \cdot 50 / 85)^{2}$ & & \\
\hline
\end{tabular}




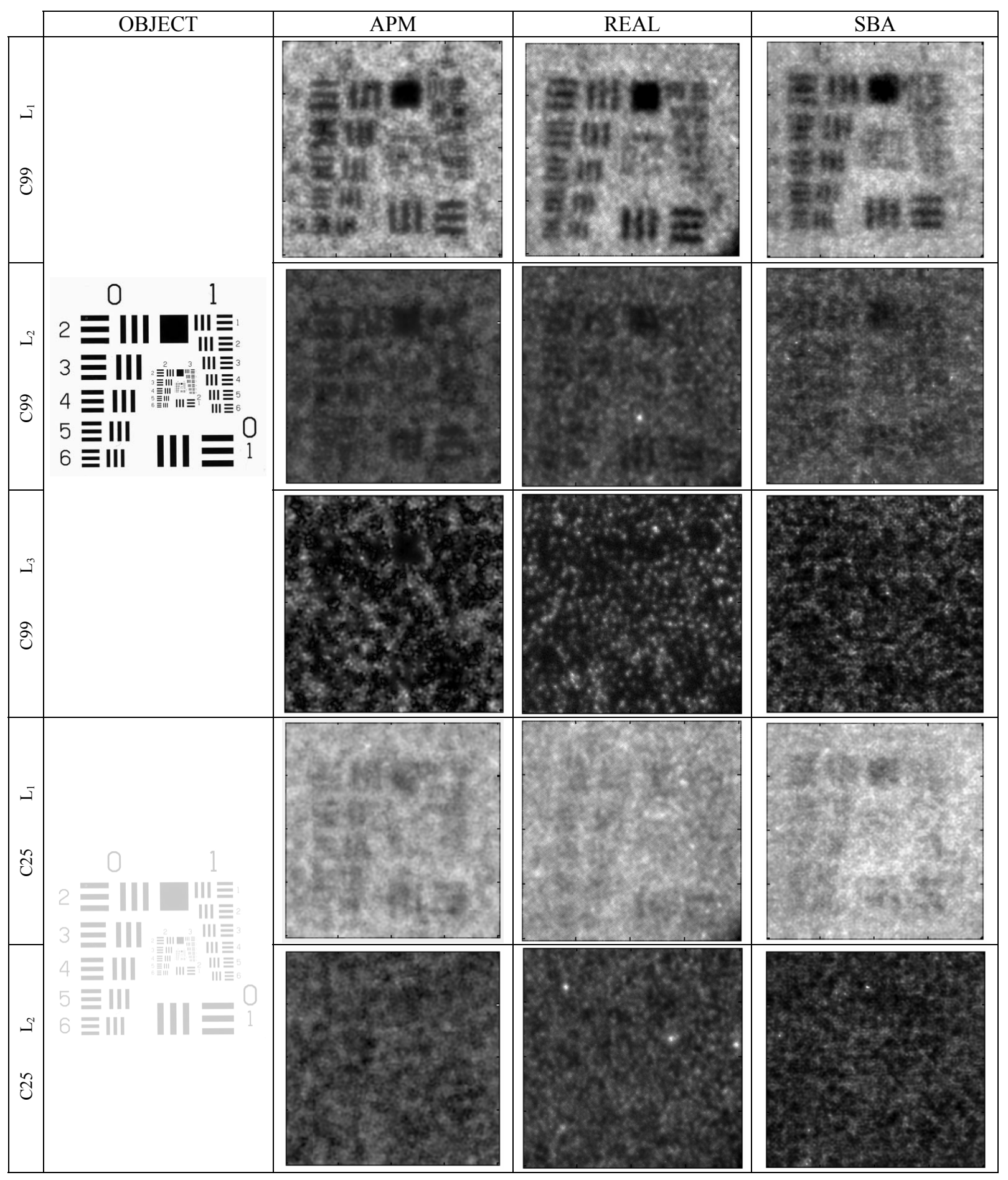

Figure 5. From top to down: USAF 1951 test chart of $99 \%$ contrast and luminances $\mathrm{L}_{1}, \mathrm{~L}_{2}$ and $\mathrm{L}_{3}$ respectively, and USAF chart of $25 \%$ contrast with luminance $\mathrm{L}_{1}$ and $\mathrm{L}_{2}$. From left to right: object, simulated image by APM, real image obtained at laboratory, and simulated image by SBA 


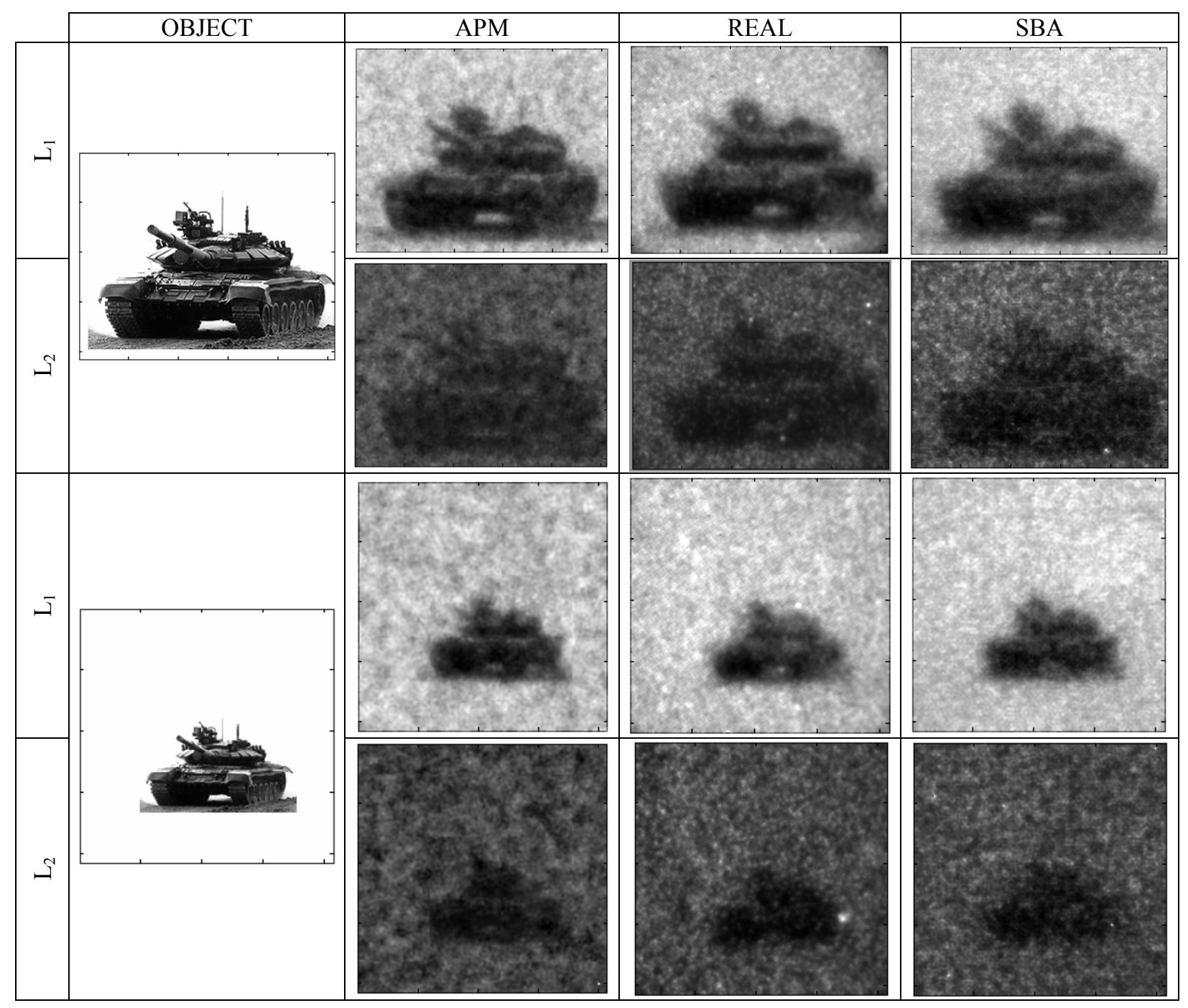

Figure 6. From top to down: tank under luminance level $\mathrm{L}_{1}$ and $\mathrm{L}_{2}$ and the same tank with half size, same luminances. From left to right: the object, the simulated image by APM, the real image obtained at laboratory, and the simulated image by SBA

\section{DISCUSSION}

The results shown in the previous section prove the ability of both image-simulation methods to generate synthetic images under conditions that are close to the performance limits of the II tube. The methods have proven to be robust, since the precision shown by simulated images is maintained when varying the simulated object, both in terms of its frequency content as well as in terms of its contrast, or the scene luminance. In this sense, figure 5 clearly illustrates how the proposed simulation methods can yield useful images capable of predicting the performance threshold of the II tube.

As shown in figure 6, these methods provide a tool to carry out a straightforward assessment of the Static Range Performance for night vision systems and turn out to be much more flexible than the indirect methods now in use. For instance, something as common as having an oblique view of the object (figure 6) invalidates most of the models used to date for the extrapolation of visibility thresholds of sights to the visibility of real objects. 


\subsection{Method suitability}

Regarding the two simulation methods employed in the present work, they have resulted to be suitable techniques. The most appropriate one depends on the particular situation. When dealing with a well-characterized II tubes, both methods turn out to be a powerful tool, both for the II tube's design and for the subsequent testing/evaluation phase. The APM turns out to be specially useful when there are no images available, and it can be helpful for system design or when choosing a II tube for a particular application. It is suitable for the simulation of image intensifier photon event counting imaging. On the other hand, when there are no reliable parameters available but one can rely on images, the SBA technique can yield a better description of the II tube's potential to be employed in other conditions (and hence, in other applications). Furthermore, when the II tube-related noise shows a peculiar statistical distribution or there is a high relative amount of background noise or high-intensity photon events are present (as cosmic rays), the SBA approach also yields better results.

\subsection{MTF}

Both methods the APM and the SBA have ended to have a high potential in terms of understanding the formation of intensified images as well as the interaction among parameters and their impact on the final image. In this sense, the effect of the MTF on the image, both due to the spatial correlation that it produces (which causes a reduction in noise) and to the blur-related image degradation, is a very important issue when working with intensified images.

The MTF is a critical input for both methods and have to be precisely measured. Even though there is no clear criteria as to how the MTF should be measured in II tubes, the various approaches available ${ }^{8}$ are all based on the measurement of the image's blur after carrying out an average to eliminate the noise component. To our understanding, the methods proposed in this work can help to design new assessment methods for the II tubes MTF capable of accounting for the interaction with noise and that can provide a better image description and a more accurate simulation.

\subsection{Video}

A lot has been written about the importance of modeling the temporal evolution of II tubes. Isolated intensified frames are seldom used. Temporal integration (carried out either by the eye or by the camera itself or even by an artificial vision device) notably improves image quality. Simulated images can be compiled as video sequences simply by putting one after the other. This is made possible as different video frames register statistically-independent events, both when recording real images and when simulating them. As long as the decay time of the exit phosphor screen (typically equal to a few ms) is much lower than the exposure time (which is in the order of tens of ms), there are very few events that end up being shared by two consecutive frames.

The main differences between simulation and reality, perceptually speaking, are much smaller for video sequences than for static images, like the ones shown in this work. For frames, the APM method seems to yield more naturally-looking images compared to those generated by SBA. This is due to the fact that SBA doesn't try to achieve an exact replica of the texture, but to provoke an equivalent visual sensation. When moving from static images to video sequences, these differences turn out to be less noticeable, since the eye is not able to perceive these differences.

\subsection{Overcoming limitations}

The methods described here can provide simulated images that could not be acquired in practice, either by unavailability of the target or by technological reasons. Furthermore, many image intensifiers are followed by an ocular which prevents them from providing high-quality intensified images. The small output pupil limits the quantity of light reaching the camera, and besides blur, introduces diffractional effects on the captured image. Simulation can overcome these limitations and can generate images that are similar to those perceived by our eye.

\subsection{High light levels}

Finally, we want to point out that neither approach has considered a fixed pattern noise (as dark spots or fiber packaging pattern), or saturation (automatic gain control activation), since the present work is focused on the range of low luminance levels where these effects are negligible. However, taking into account that these effects have a big impact for high luminance levels, it could be an interesting subject for future research. 


\section{CONCLUSIONS}

Two methods for the simulation of intensified images have been developed within the context of the present work. Both techniques yield good results in terms of visual comparison. For want of a more rigorous study, these preliminary results suggest that both methods can accurately predict discrimination thresholds for II tubes for any given combination of spatial (or angular) frequency, contrast of luminance of the object.

Specifically, these tools can produce a more straightforward measurement of the static range performance of night vision systems based on intensified images.

Finally, it is worth to highlight that, unlike other existing models, our approach is quantitative and based only on wellknown or measurable parameters and yields simulated images which remain faithful to reality even in situations that are close to the performance limits of the II tube.

\section{ACKNOWLEDGEMENTS}

The authors gratefully acknowledge the financial support provided by the Subdirección General de Tecnología y Centros of the Spanish Ministry of Defence.

\section{REFERENCES}

1. Lucien M. Biberman (editor), Electro-Optical Imaging: System Performance and Modeling, SPIE Press, USA 2000.

2. I. Csorba, Image tubes, Howard W. Sams, New York NY, 1985

3. K. R. Fowler, "Automatic Gain Control for Image-Intensified Camera", IEEE Transactions on Instrumentation and Measurement, 53 (4), 1057-1064, 2004.

4. S. E. Moran, B. L. Ulich, W. P. Elkins, R. L. Strittmatter, and M. J. DeWeert. "Intensified CCD (ICCD) dynamic range and noise performance." SPIE Vol. 3173 Ultrahigh and High-Speed Photography and Image-Based Motion Measurement, pp. 430-457, 1997.

5. A. Frenkel, M. A. Sartor, and M. S. Wlodawski. "Photon-noise-limited operation of intensified CCD cameras". Applied Optics, 36 (22), 5288-5297, 1997.

6. P. J. Thomas, R. S. Allison, P. Carr, E. Shen, S. Jennings, T. Macuda, G. Craig, R. Hornsey, "Physical Modeling and Characterization of the Halo Phenomenon in Night Vision Goggles", SPIE vol. 5800 Spaceborn Sensors II, 2005.

7. E. J. Ientilucci, S. D. Brown, J. R. Schott, R. V. Raqueno, "Multisprectral Simulation Environment for Modeling lowlight-level Sensor System", SPIE Conference on Image Intensifiers and Applications, San Diego, CA, USA, 19-24 07 1998, vol. 3434, pp. 10-19.

8. S. Ortiz, D. Otaduy, C. Dorronsoro, "Optimum parameters in image intensifier MTF measurements." SPIE vol. 5612 Conference on Defence and Security, pp. 382-391, 2004.

9. J. Portilla, R. Navarro, O. Nestares, and A. Tabernero. "Texture synthesis-by-analysis method based on a multiscale early-vision model.” Optical Engineering, 35(8):2403-2417, 1996.

10. O. Nestares, R. Navarro, J. Portilla, and A. Tabernero. "Efficient spatial-domain implementation of a multiscale image representation based on Gabor functions". Journal of Electronic Imaging, 7(1):166--173, 1998. 